\title{
Americas' Geospatial Response to COVID-19
}

\author{
Rosario Casanova, Paloma Merodio Gómez, Álvaro Monett Hernández and \\ Andrea Ramírez Santiago
}

\begin{abstract}
In the Americas region, the Regional Committee of the United Nations for Global Geospatial Information Management (UN-GGIM: Americas) in a joint work with the Economic Commission for Latin America and the Caribbean, have been facilitating exchange of good practices and monitoring geospatial tools to respond to the pandemic. This exchange has conveyed an analysis of the different institutional arrangements, data management and technological approaches taken by countries in the region to control the outspread of COVID-19. This chapter provides an overview of the regional geospatial response to COVID-19. We analyze the implementation and use of UNGGIM global frameworks in the geospatial response to COVID-19. Through the regional meetings and consultations carried out in the context of the pandemic, we have been able to verify that countries, to a greater or lesser extent, have knowledge on methods and geospatial tools to face critical situations such as the COVID-19 pandemic. However, some gaps and challenges have also been identified that must be addressed. From a regional perspective, we encourage countries to take full advantage of these guiding frameworks, through a collective and collaborative approach.
\end{abstract}

\subsection{Introduction}

In December 2019, a new virus called SARSCoV-2 causing severe acute respiratory syndrome coronavirus disease (COVID-19) emerged in Wuhan, China, and rapidly spread to other parts of the world [1]. March 11, 2020, the World Health Organization (WHO) officially declared the COVID-19 outbreak a pandemic [2]. The disease is characterized by a long incubation period, high infectivity, and difficulty in detection, which has contributed to the rapid outbreak and development of the epidemic [3].

This situation has required that experts in different areas like doctors, disease trackers, modelers, logisticians, and supply chain experts are designing and implementing measures to stop the transmission and spread of the virus. In this way, it is essential for the exchange of information and the development of tools, to deliver data in real time on websites and via messaging networks, identification of locations to establish additional hospitals, quarantine bases and virus testing locations, and effective communication on the situation.

The COVID-19 pandemic is full of unknowns, and many of them have a spatial dimension [3], in this way, GIS has become a vital tool in analyzing and visualizing the spread of COVID-19 [4]. Modern GIS technologies center around web-based tools, improved data sharing and real time information to support critical decision-making, an example of these are online dashboards have been extremely popular to sharing and understanding the spread of COVID-19, since they offer accessible information to people around the world, improves data transparency and helps authorities 
disseminate information [1]. The most notable example is the online dashboard developed by John Hopkins University Centre for Systems Science and Engineering [5].

In this way, this chapter provides an overview of the regional use of geospatial data and GIS tools to support the response measures and manage the containment of COVID-19, based on the outputs of the Virtual Geospatial Summit, the webinar "COVID-19: Strategies for a Geospatial Response in the Americas" and the regional questionnaire on geospatial support to COVID-19 in the Americas. Furthermore, we analyze the role and implementation of UNGGIM global frameworks in the geospatial response to COVID-19.

\section{Available Global Frameworks to Support the Geospatial Response to COVID-19}

The geospatial response to COVID-19 at country level can be supported by sound global guidelines provided by the United Nations Committee of Experts on Global Geospatial Information Management (UNGGIM [6]). This committee has the objective to make joint decisions and establish directions on the use of geospatial information within national and global policy frameworks.

In this chapter, we will show how the geospatial response to COVID-19 -being conducted by member states is transiting to the alignment with these frameworks and how these frameworks can help so that .an improved geospatial response to this emergency and others that may occur in the future is sustainable.

The first one is the Integrated Geospatial Information Framework (IGIF [7]) that provides a guide for developing, integrating, strengthening, and maximizing geospatial information management and related resources in all countries [8]. The IGIF has been proven to be integrative and of practical implementation. Any of the nine IGIF strategic pathways have been implemented by member countries in the Americas, by establishing institutional arrangements and governance agreements, referencing policies to protect privacy and confidentiality of data, promoting partnerships between stakeholders from different sectors (public, private, and academy), leveraging innovation to deliver better technological platforms, and creating communication tools to reach the wide spectrum of users that need this geospatial resources for different purposes.

The second framework endorsed by UNGGIM and the Statistical commission of the United Nations Statistics Division that has proven to be usefully implemented to monitor COVID-19 is the Global Statistical and Geospatial Framework (GSGF [9]) which enables a range of data to be integrated from both statistical and geospatial communities and, through the application of its five Principles and supporting key elements, permits the production of harmonized and standardized geospatially enabled statistical data. The resulting data can then be integrated with statistical, geospatial, and other information to inform and facilitate data-driven and evidence-based decision making. The integration of statistics and geospatial has been vital in this pandemic. Sociodemographic variables such as age, health status, have been analyzed even at block level to detect vulnerable populations.

And the third one is the Strategic Framework on Geospatial Information and Services for Disasters [10], elaborated by the UN-GGIM Working Group on this topic, which aims to bring all stakeholders and partners involved in Disaster Risk Reduction and/or Emergency Management together to ensure that quality geospatial information and services are available and accessible in a timely and coordinated way to support decision-making and operations within and across all sectors and phases of disaster risk management. The strategic framework draws from the principles included in the Sendai Framework for Disaster Risk Reduction [11]; the UN General Assembly resolution on international cooperation on humanitarian assistance in the field of natural disasters, from relief to development; the 2030 Agenda for Sustainable Development; and the UN-GGIM Global Statistical Geospatial Framework [9]. 


\subsection{Overview On the Regional Geospatial Response to COVID-19}

As reported in the abstract of this article in a joint effort between the United Nations Regional Committee for Global Geospatial Information Management (UN-GGIM: Americas) and the Economic Commission for Latin America and the Caribbean (ECLAC [12] with the support of the Secretariat of UN-GGIM at a global level, two regional webinars and a regional consultation on regarding geospatial response to COVID-19 were conducted, in order to collect information and share experiences and knowledge from different approaches ranging from institutional to technological.

In order for geospatial data users and providers across governments, the private sector, academia, students and the general public could know and shared how the global community of geospatial scientists have been leveraging geospatial, Earth Observation and statistical data, creating innovative tools to support response measures and manage the containment of COVID-19, was made the Virtual Geospatial Summit 2020 under the theme "GIS Response to COVID-19", in which they showed some of the geospatial tools that have been developed in different countries including the Americas region, as well as the good practices that have been implemented, including the challenges in collecting geospatial health data.

The seminar COVID-19: Strategies for a Geospatial Response in the Americas was held on May 15, 2020, facilitated by UN-ECLAC and UN-GGIM: Americas. Through this activity an enriched exchange of experiences in Member States on how they have met the challenge of COVID-19 was conducted, reviewing progresses, and identifying the challenges being faced. Demonstrations on developing national dashboards and tools were also shared, highlighting data needs and available resources. The seminar helped the discussion on how to optimize resources to respond in the short and medium-term impact of COVID-19, while preparing for the long-term implications of public health and safety crises or emergencies.

The regional consultation was carried out by UN-GGIM: Americas and ECLAC to promote dissemination actions and exchange of practices and experiences around the use of geospatial information, to support the management of the COVID-19 pandemic.

In this consultation, information was collected from national geospatial data infrastructures or from national cartographic agencies in collaboration with other public bodies. Information regarding data integration, development of indicators and implementation of platforms, among others was reported. The identified use cases and good practices were disseminated on the UN-GGIM: Americas website [13], and on the COVID-19 Observatory [14] in Latin America and the Caribbean.

\section{Overview On the Regional Geospatial Support}

The following section presents the response approach, from different countries in the region, regarding institutional aspects and the use of geospatial data.

\section{Governance and Institutions}

A central component of geospatial support for disaster management is governance. This is established by the UN-GGIM Strategic Framework on Geospatial Information and Services for Disasters, assigning institutional agreements, collaboration, and coordination a fundamental role. In this context, through this consultation it was possible to identify various forms of governance and participation in the countries that responded to the regional consultation, basically through the formation of multisectoral work teams to support decision-makers and the entities in charge of disaster management.

In order to articulate national organizations and to make geospatial information available for decision-making, several countries have activated protocols to support to the entities in charge of disaster management, like the National Emergency Response System in Jamaica, the Emergency Operations Center in Sint Maarten or the National Risk Management System in Honduras. These protocols organize the collection of spatial information, the integration of statistical and geospatial data and the way in which this information is processed to help the decision making. 


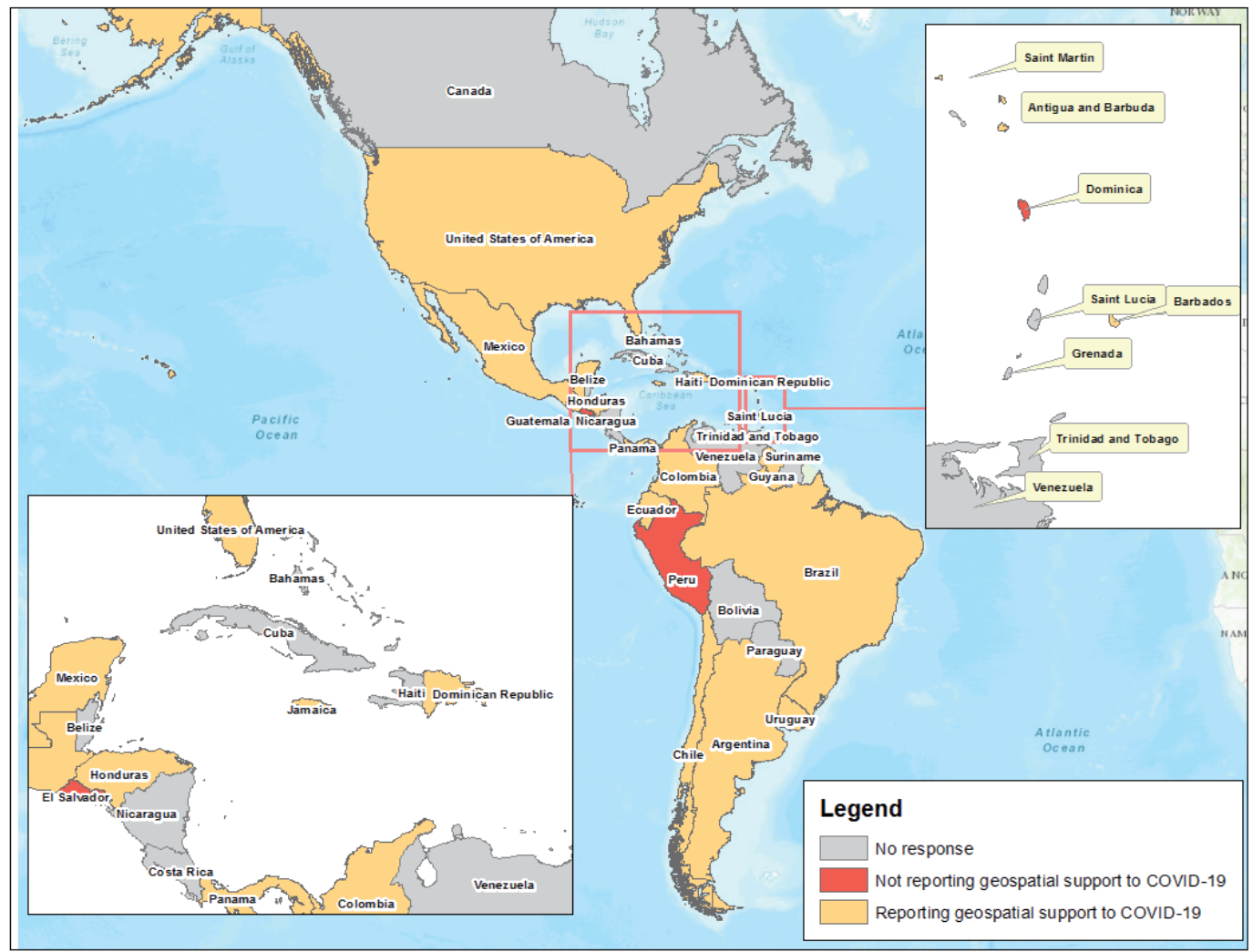

\section{FIGURE 19.1}

Map representing the geospatial response to COVID-19 of Americas countries. Source: Regional consultation carried out by UN-GGIM: Americas-ECLAC Regional consultation, 2020.

Other frequent institutional arrangement observed in the region is the creation of special working committees and/or groups to assist the national geospatial response to the pandemic. This allows to collect information from various sources, keep the inter-institutional communication permanently opened and generate added value products helping to implement logistical actions and carry out territorial analysis regarding the advance of the pandemic. These working groups in some cases also examine political issues affecting employee activities, travel, and public events, among others.

In this regional overview, the institutions that most frequently participate in geospatial support are the ministries and institutes of statistics and geography of each country. An example of the above is Mexico, where statistical data on COVID-19 is provided by the Ministry of Health, and then these are processed by the National Institute of Statistics and Geography (INEGI), to be displayed on platforms for presentation to the public; Chile follows a similar process, but in this case it is through the country's Spatial Data Infrastructure (IDE-Chile) that collects and disseminates the information provided by the different associated Ministries or Institutions.

Following the foregoing, in many countries emergency systems or institutions are the first access route in geospatial support, an example of this is Uruguay, where the National Emergency System (SINAE) is the body in charge of being in contact with all institutions from which you can receive information; In Antigua and Barbuda, the National Disaster Office (NODS) performs a similar function, coordinating the collaboration of geospatial information from different Ministries or offices such as the statistical office. Also, national / civil defense institutions are organizations that have been mentioned as organizations that deliver information to the coordinating entity, as is the case of Guyana, Barbados, the Dominican Republic and Jamaica. Finally, to a lesser extent, agencies, private entities, or foundations are independent organizations that have valuable information useful 
for the country, for which they work collaboratively. This is the case of Brazil, where data provided by the Oswaldo Cruz Foundation is disseminated; or in the Dominican Republic, where support is obtained from private geomatic companies.

The integration of statistical and geospatial information has been made explicit through the examples mentioned above, where countries are using geocoded health and population data referenced to a specific location and the information is being displayed in geospatial dashboards. This use of fundamental geospatial infrastructure and geocoding in a data management environment, using common geographies for the dissemination of statistics in an accessible and usable platform is the core of the five principals of the GSGF [15].

\section{Data and Technology}

From the collected information through the different activities and consultations carried out in the region, it was possible to realize that the most frequent technological resources being utilized to disseminate COVID-19 statistics, are geospatial dashboards. Secondly, interactive websites with maps, charts and dynamic statistics. Most countries are using GIS to disseminate statistics related to confirmed cases, active cases, recovered cases, and deaths, at different levels of disaggregation, such as political-administrative divisions, gender, or age groups. A first group of countries report that they are using GIS to prepare distribution maps, networks and flows of people to access health services, or other services that offer basic goods. Others have informed to be carrying out spatial analysis of vulnerable groups, such as the elderly, the chronically ill or areas of high population density. There are also cases of mapping households with confirmed cases, quarantined areas and isolation centers, as well as constant monitoring of confirmed and suspected cases.

In the case of the regional consultation, it was informed that, from a total of 20 countries that answered the questionnaire, fifty percent declared that they had public access platforms for the dissemination of data regarding COVID-19. In general terms, the information is disaggregated to the second or third hierarchical level of the political-administrative division and the topics represented are diverse: confirmed cases, examinations, intensive care patients and deceased patients, vulnerable population disaggregated by age ranges, information on employability, poverty and population at risk, tests carried out and results, among others.

Regarding some specific country examples in the use of national platforms and dashboards, the National Institute of Statistics and Geography of Mexico developed a geoportal called "Analytical Visualizer for COVID-19", which allows information on COVID-19 how number of confirmed, negative, suspected cases and deaths to be seen at the municipal and state levels. Information that is aligned to the information issued by the Ministry of Health.

In addition to this information, the displayed integrates information of others 8 themes: population, ethnicity educational characteristics, economic characteristics, health services, households, hospital infrastructure and deaths due to various registered conditions 2018. Each one of the topics has its corresponding indicators for a total of 28 , some of the indicators that can be viewed are density (inhabitants per square kilometer), total population, population over 60 years of age, population affiliated with health services, average number of occupants per dwelling, dwellings that do not have piped water and risk diseases.

Other interesting experience is that conducted collaboratively between the National Planning Department, the Institute of Technological Evaluation in Health and the National Administrative Department of Statistics (DANE) of Colombia, which collaborated with the Ministry of Health and Social Protection and the National Institute of Health to provide statistical information and build tools ${ }^{1}$ that facilitate senior government to make decisions with greater certainty for emergency response caused by COVID-19.

In this case, the demographic characteristics of the population and their conditions of health helped to determine who may have more complications in case of getting COVID-19. This, by taking into account, among others considerations, the identified epidemiological criteria, based on information from the 2018 National Census of Population and Housing, administrative records of the National Identification File, the Civil Birth Registry, the National Registry of Marital Status, the Unique Database of Health Affiliation and the individual records of provision of Health services.

\footnotetext{
${ }^{1}$ Reference: http://visor01.dane.gov.co/visor-vulnerabilidad/
} 

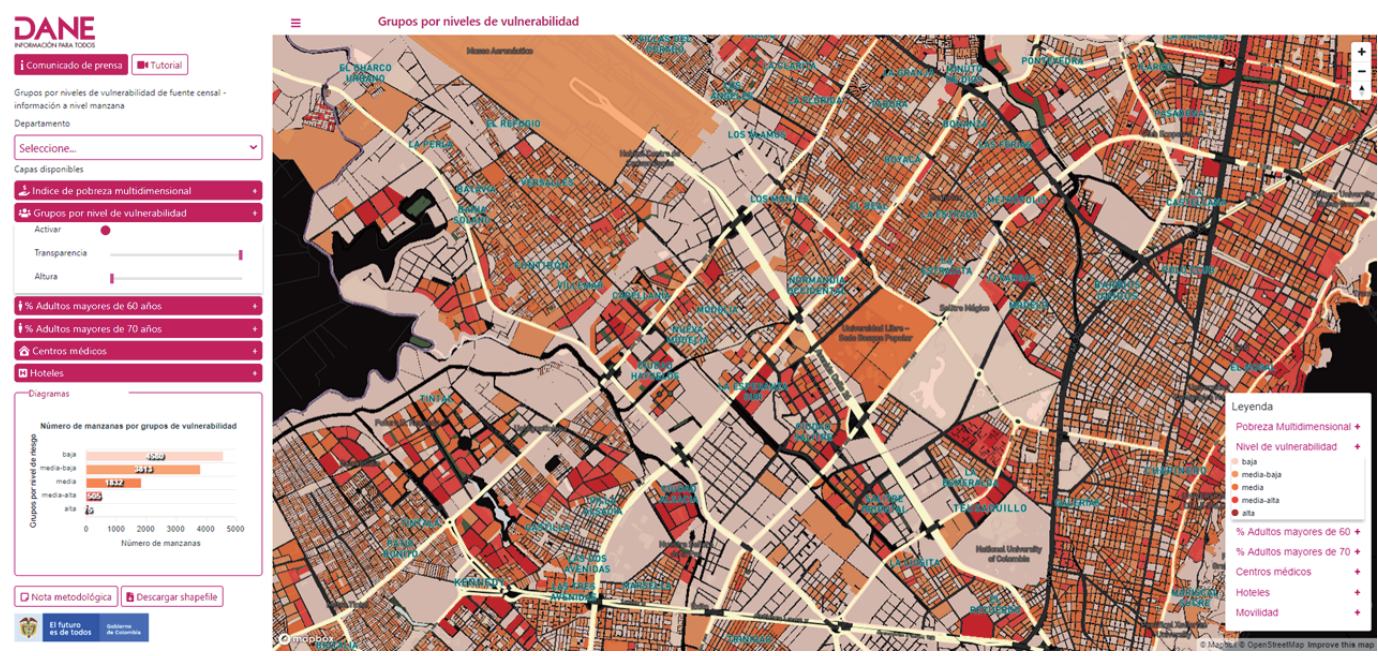

\section{FIGURE 19.2}

Viewer of vulnerability levels in Colombia Source: National Administrative Department of Statistics of the Republic of Colombia, 2020.

In the Caribbean subregion, it can be mentioned the work carried out by the GIS unit of the Survey and Mapping Division, which has been providing geospatial support to the National Emergency Operation Center, mainly through the development of GIS solutions and data gathering. This work has been conducted in coordination and collaboration with the National Office of Disaster Services, the Ministry of Health, Wellness and the Environment, the Central Board of Health and the Statistics Division. As a result, a geospatial hub is in the public domain to disseminate data on the timeline of cases and basic associated information, primarily focused on the local level, but complemented by a section regarding regional response efforts. Internally, a monitoring dashboard is still being setup to effectively manage the operations and coordination between all stakeholders.

In Central America, the National Geographic Institute Tommy Guardia (IGNTG) of Panamá formed a work team to support with cartographic information and mapping, two relevant government initiatives to confront COVID-19. The first on is the plan "Protégete Panamá" led by the Ministry of Health in collaboration with the Social Security Fund, WHO experts, and national and international Health experts. The second one is the "Solidarity Plan", in charge of the Economic Advisory Council led by the Ministry of the Presidency and integrated by several institutions. Both programs seek to mitigate the impact of the pandemic and guarantee that Panamanians affected by the health crisis can obtain essential products.

The IGNTG, leading the Panamanian Spatial Data Infrastructure, has facilitated data cooperation to integrate information from different sources, for example, on educational centers location (provided by the Ministry of Education) and a wide range of statistical data made available by the Institute of Statistics and Censuses, which has been combined with other geospatial information.

The committee prepared a special map of the republic with presidential indications for the Solidarity Plan for food delivery logistics and solidarity bonds to affected families: Route maps for garbage collection; Maps to assist aid distribution and logistics for municipalities; Statistical information to generate information layers of housing areas for people over 60 years, beds by province, chronic diseases, location of health centers and hospitals; Maps for aircraft landing areas and secondary collection centers. 


\subsection{Gaps and Challenges}

The outcomes from the regional consultation show that countries have been able to capitalize the power of geospatial tool to respond to COVID-19. Nevertheless, challenges persist in the region and further work needs to be done in order to have an integrated geospatial response and to be prepared for future disasters.

- The most urgent gap detected by the regional encounters and consultations is related to data accessibility. To respond geospatially to the pandemic countries have faced challenges in: accessing updated real time data; lack of access to quality data and satellite images; unavailability of disaggregated fundamental data at different levels (political-administrative divisions, sex, age, etc.). Moreover the low interoperability between statistical and geospatial data, the absence of regulation regarding the use of information, and the need to create a national address systems are the most frequent challenges among the countries that responded the questionnaire conducted by ECLAC/UN: GGIM-Americas.

- Farther, there is an urgent need to improve the GIS technical capacities of current personnel. Some of the weaknesses, that have been exposed in this pandemic, refer to the lack of technical and professional resources available to make appropriate use of geospatial data, as well as lack of knowledge about the accessibility to free geographic data and applications.

- Raising awareness of authorities regarding the importance of GIS can promote its effectiveness in capturing, analyzing and disseminating spatial information. Countries recognize the challenge to support software financing and human resources. Finally, it is important to have an appropriate Spatial Data Infrastructure (SDI) that allows them to collect data from the different institutions, to have a cadaster and organize national information.

On the other hand, two major advancements identified in the regional consultation are related to dissemination geospatial tools and partnerships.

- The urgent need to access high quality data has been enabled through geospatial dissemination tools. The public visibility of geographic data, gained during this pandemic, has made geographic information take on unexpected relevance. Fact that opens some opportunities and challenges for the geomatic community. Moments in which the relevance of geographic data, as a tool for processing and making analysis, is an unquestionable fact open opportunity to promote and raise awareness of the importance of having upgraded and good data quality of the information. Data that should be accessible at the national level and acquired with the specific needs of each country. An aspect that should be included, in the current agenda, refers to the use of geospatial information in support of the new "normality", in which sustained physical distance is the only way to minimize the effects of the pandemic. Now it is time to move from response to recovery and reopening. Once, commercial life, educational centers, recreational spaces, etc. have been reestablished, the relative distance between the inhabitants begins to take on a leading role in the new way of life. And it that place is where geomatic tools have a lot to contribute.

- The wealth of professional exchanges carried out in these times has been one of the most outstanding aspects of this pandemic. As stated in the ninth pathway of the IGIF, cross-sector and interdisciplinary cooperation, coordination and collaboration with all levels of government, the geospatial industry, private sector, academia, and the international community is a premise to developing and sustaining an enduring response to disasters [8]. The collaboration and partnership generated by various professionals, from different countries, has resulted in the use of good practices that have no frontiers and are being applied in different parts of the continent. As geospatial professionals, practitioners and stakeholders strategic actions reinforcing the power of collaboration and true humanity. 


\subsection{Conclusions}

In the previous sections, valuable experiences in the use of geospatial information to support the response to COVID-19 in countries of the region have been described,. Various institutional arrangements to coordinate the actions of the different national actors, to establish the links between geospatial agencies, ministries of health, and other relevant actors, with the offices in charge of emergency management. We also provided an overview of methodologies for the integration and analysis of geospatial data and its dissemination through accessible platforms for decision-makers and citizens.

Through the regional meetings and consultations carried out in the context of the pandemic, we have been able to verify that the countries, to a greater or lesser extent, have knowledge on methods and geospatial tools to face critical situations such as the COVID-19 pandemic. However, some gaps and challenges have also been identified that must be addressed, regarding to interoperability, data access policies, higher levels of disaggregation, capacity building, increased awareness at the level of authorities and availability of greater financing, among other.

Considering the above, in the region of the Americas there are strengths which support future crises of this or another nature. We also have weaknesses and challenges that open the way to strengthen our geospatial response. The crucial question then is, what should we do to capitalize our strengths, address gaps and achieve a comprehensive and sustainable geospatial response over time?

From UN-GGIM: Americas and ECLAC, we recognize a great opportunity in the new guidelines delivered by the working agenda of the UN-GGIM Initiative, and the need to bring down global frameworks in the countries of the region. These frameworks would make it possible to take advantage of and strengthen the response that we have today, provide cross-cutting work elements to the countries' management in geospatial matters, and prepare roadmaps with concrete actions to cover the existing gaps.

The Integrated Geospatial Information Framework (IGIF) and the Implementation Guide for its nine strategic pathways -governance and institutions, legal and policy issues, financing, geospatial data, innovation, standards, partnerships, education/capacity building, and communicationexhaustively and didactically provides a vision of what actions can be carried out to strengthen geospatial information management.

In particular, the Implementation Guide provides valuable guidance to address the five priorities of action of the Strategic Framework on Geospatial Information and Services for Disasters, which are connected and aligned with the IGIF's strategic pathways. For example, for the Disaster Framework Governance action priority, the IGIF explains how to establish working groups, define strategies, develop action plans, and monitor their progress, among other.

The same applies to other priorities of action such as data management, where the IGIF implementation guide suggests on the application of inventories (in this case to support disasters management), the development of geospatial data profiles, the analysis of gaps, the formulation of roadmaps for thematic data, and the generation of guidelines for the maintenance and custody of data, among others.

On the other hand, the Global Statistical Geospatial Framework (GSGF) provides tools for the territorial disaggregation of data, a fundamental requirement to have accurate diagnoses and to effectively guide the activation of alerts and decision-making for the management and recovery of this health, social and economic crisis. The GSGF highlights the importance of geocoding processes and the fundamental geospatial dataset that support it, for example, geo-referenced postal addresses, buildings, cadastral parcels, and other highly granular data.

From the regional level, we encourage countries to take full advantage of these guiding frameworks, through a collective and collaborative review among all public, private and academic actors, and then put them into operation on the basis of institutional agreements, intersectoral alliances, capacity building plans, and sustainable communication mechanisms over time. 


\section{References}

[1] Maged N Kamel Boulos and Estella M Geraghty. Geographical tracking and mapping of coronavirus disease covid-19/severe acute respiratory syndrome coronavirus 2 (sars-cov-2) epidemic and associated events around the world: how 21st century gis technologies are supporting the global fight against outbreaks and epidemics, 2020 .

[2] World Health Organization. World health organization website, 2020.

[3] Ivan Franch-Pardo, Brian M Napoletano, Fernando Rosete-Verges, and Lawal Billa. Spatial analysis and gis in the study of covid-19. a review. Science of The Total Environment, page 140033, 2020.

[4] Abolfazl Mollalo, Behzad Vahedi, and Kiara M Rivera. Gis-based spatial modeling of covid-19 incidence rate in the continental united states. Science of The Total Environment, page 138884, 2020.

[5] Johns Hopkins University and Medicine. Johns hopkins coronavirus resource center website, 2020.

[6] UN-GGIM: Américas. Un-ggim: Américas website, 2020.

[7] United Nations. Integrated geospatial information framework (igif), 2020.

[8] United Nations-Statistics Division and World Bank. Integrated geospatial information framework: A strategic guide to develop and strengthen national geospatial information management part 1: Overarching strategic framework, 2018.

[9] United Nations-Statistics Division. Global statistical geospatial framework, 2019.

[10] United Nations-Committee of Experts on Global Geospatial Information Management Working Group on Geospatial Information and Services for Disasters. Strategic framework on geospatial information and services for disasters, 2017.

[11] United Nations Office for Disaster Risk Reduction. Sendai framework for disaster risk reduction, 2015.

[12] ECLAC United Nations. Economic commission for latin america and the caribbean website, 2020.

[13] UN-GGIM: Americas. Un-ggim: Americas website, 2020.

[14] ECLAC United Nations. Observatorio covid-19 en américa latina y el caribe: Impacto económico y social, 2020.

[15] United Nations Expert Group on the Integration of Statistical and Geospatial Information. The gsgf implementation guide, 2019. 
$\Longrightarrow$ Taylor \& Francis Taylor \& Francis Group

http://taylorandfrancis.com 